\title{
Relação entre a deformação residual longitudinal com crescimento e propriedades da madeira de espécies de Eucalyptus
}

\author{
Relation of residual longitudinal strain with tree \\ growth and wood properties of Eucalyptus species
}

Rafael Leite Braz ${ }^{1}$, Jorge Luis Monteiro de Matos², Márcio Pereira da Rocha ${ }^{2}$ e José Guilherme Prata ${ }^{2}$

\begin{abstract}
Resumo
O presente estudo teve como objetivo avaliar a relação da deformação residual longitudinal (DRL) decorrentes das tensões de crescimento com as variáveis de crescimento das árvores e as propriedades físicas e mecânicas da madeira. Foram utilizadas as espécies de $E$. dunnii, e $E$. grandis com 40, 52 e 64 meses e o E. saligna com idades de $28,40,52$ meses, destinados a geração de produtos sólidos. Os níveis de tensão de crescimento foram mensurados indiretamente pelo método do CIRAD-Forêt. As propriedades físicas e mecânicas da madeira foram obtidas de acordo com as normas da COPANT (1972a). As correlações entre a DRL e as variáveis de crescimento das árvores, propriedades físicas e mecânicas apresentaram uma alta dispersão, apresentando a madeira aos 52 meses de idade uma correlação mais evidente entre as variáveis investigadas. Dessa forma, programas de melhoramento genético florestal, associados aos incrementos de novas práticas silviculturais devem ser direcionados para a produção de madeira com características desejáveis para a obtenção da madeira sólida, com baixa propensão das tensões de crescimento.
\end{abstract}

Palavras-chave: Tensões de crescimento, qualidade da madeira, produtos sólidos.

\begin{abstract}
This study aimed to assess the relationship of longitudinal residual strain due to growth stresses with tree growth variables and the physical and mechanical properties of wood. The species studied were $E$. dunnii and $E$. grandis, both with 40, 52 and 64 months of age and $E$. saligna with ages 28, 40, 52 months of age for solid wood production. The growth stress levels were measured indirectly through the CIRAD-Forêt method. The physical and mechanical properties of wood were obtained in accordance with the standards of COPANT (1972a). Correlations among longitudinal residual strain with tree growth variables and physical and mechanical properties showed a high dispersion; wood at 52 months of age exhibited a more obvious correlation among investigated variables. Thus, forest breeding programs, associated with increments of new silvicultural practices should be directed to the production of wood with desirable characteristics for obtaining the solid wood with low propensity for growth stress.
\end{abstract}

Keywords: Growth stress, wood quality, solid products.

\section{INTRODUÇÃO}

Diversas espécies do gênero Eucalyptus, ao longo dos anos passaram a ser plantadas como alternativa de produção para suprir demandas das indústrias dos setores florestais e madeireiros, uma vez que podem ser empregadas e utilizadas em diferentes situações, além de possuírem alta capacidade produtiva.

O grande potencial do eucalipto para usos nobres está relacionado principalmente a sua diversidade de espécies, alta capacidade de geração de clones e híbridos. Esta diversidade permite a introdução do gênero em programas de melhoramento genético, de condução das florestas, de manejo e uso de tecnologias adequadas de processamento e usinagem que se mostrem eficientes na solução ou redução de defeitos, tais como rachaduras de topo e empenamento, em toras e tábuas. Defeito em grande parte, devido às tensões de crescimento o que contribui para a depreciação do valor da madeira. (TRUGILHO et al., 2006)

\footnotetext{
1Professor Adjunto do Departamento de Ciência Florestal. UFPE - Universidade Federal Rural de Pernambuco. Dois Irmãos - 52171900 - Recife, PE, Brasil. E-mail: rlbraz.ufrpe@gmail.com.

${ }^{2}$ Professor Associado do Departamento de Engenharia e Tecnologia Florestal. UFPR - Universidade Federal do Paraná. Rua Lothario Meissner, 620 - Jardim Botânico - Caixa-postal: 19061 - 80210-170 - Curitiba, PR, Brasil. E-mail: jmatos@ufpr.br; mprocha@ufpr.br; prata@ufpr.br.
}

Sci. For., Piracicaba, v. 44, n. 111, p. 629-640, set. 2016 DOI: dx.doi.org/10.18671/scifor.v44n111.09 
As tensões de crescimento e sua magnitude podem ser determinadas a partir da medição da deformação residual longitudinal (DRL), que consiste na medição indireta da tensão, quando liberada no sentido longitudinal das árvores. Tais valores da DRL, quando associados a outras propriedades da madeira, podem contribuir para estabelecer parâmetros que gerem modelos para estimar as tensões de crescimento e indicar fatores que colaboram para a seleção de materiais com menor propensão a defeitos.

Uma grande dificuldade para o uso de madeira de eucalipto na produção de madeira serrada, é o aparecimento de rachaduras e empenamento em decorrência das tensões (PÁDUA et al., 2004). Os mesmos autores afirmam que as tensões são as principais responsáveis pelo baixo rendimento no processamento da madeira de eucalipto, devido à redução das dimensões das peças.

O crescente emprego da madeira do gênero Eucalyptus para produtos sólidos desencadeou diversas questões sobre níveis e variações das tensões de crescimento, buscando a produção da madeira com qualidade superior voltada para o mercado de produtos sólido, agregando valor ao produto final. Segundo Trugilho et al. (2005) e Beltrame (2012), a avaliação das tensões de crescimento tornou-se uma ferramenta bastante útil na seleção e classificação de genótipos, permite ainda a comparação entre árvores e o acompanhamento das alterações que possam ocorrer nos diferentes tratamentos para redução e, consequentemente, para seleção das espécies com baixas tensões de crescimento, visando estudos genéticos.

Nicholson et al. (1975) encontraram fortes correlações entre as tensões de crescimento e algumas propriedades físicas e mecânicas da madeira. Clair et al. (2003) também encontraram relações significativas entre as tensões de crescimento e as propriedades físicas e mecânicas da madeira, especialmente na direção longitudinal.

O conhecimento da relação das tensões de crescimento e outras propriedades da madeira são essenciais para a avaliação da qualidade da madeira serrada destinada a produção de produtos sólidos de alto valor agregado, além dos recursos que podem ser utilizados a fim de melhorar o seu processamento e uso. Neste contexto, o objetivo do presente estudo foi verificar relação entre a DRL e as variáveis de crescimento das árvores, e as propriedades físicas e mecânicas da madeira das espécies de E. dunnii, E. grandis e o E. saligna em diferentes idades.

\section{MATERIAL E MÉTODOS}

O material utilizado neste estudo foi proveniente de plantios comerciais de Eucalyptus spp., implantados com espaçamento de $5 \mathrm{~m}$ (entre linhas) x 2,8 m (linha), pertencentes à empresa GRANFLOR destinados à produção de madeira para geração de produtos sólidos, coletados no munícipio de Rosário do Sul, Estado do Rio Grande do Sul.

O munícipio situa-se a $30^{\circ} 15^{\prime} 28^{\prime \prime}$ sul de latitude, $54^{\circ} 54^{\prime} 50^{\prime \prime}$ oeste de longitude e altitude de $125 \mathrm{~m}$. A região em geral, caracteriza-se por apresentar uma precipitação média anual ente 1300 a $1600 \mathrm{~mm}$ e uma temperatura média anual superior a $32^{\circ} \mathrm{C}$. De acordo com a classificação climática de Köppen, o clima é do tipo subtropical úmido, com verões quentes e invernos frios (cfa). Considerando a Classificação da Vegetação Brasileira, a área corresponde às formações de estepe do bioma Pampa (IBGE, 2004).

Na Tabela 1 consta as espécies utilizadas, bem como o número de árvores que foram amostradas e as respectivas idades, num total de 92 amostras.

Tabela 1. Amostragem do material.

Table 1. Sampling of the material.

\begin{tabular}{lcc}
\hline Espécies & Idade (meses) & $N^{0}$ de árvores \\
\hline \multirow{2}{*}{ E. dunnii } & 65 & 16 \\
& 52 & 5 \\
\hline \multirow{2}{*}{ E. grandis } & 40 & 10 \\
& 65 & 16 \\
& 52 & 5 \\
E. saligna & 40 & 10 \\
\hline
\end{tabular}




\section{Variáveis de crescimento das árvores}

Com auxílio de uma fita métrica, mediu-se a circunferência à altura do peito (CAP), cujos dados foram transformados em diâmetro à altura do peito (DAP). A altura total (Ht) das árvores foi medida com o Hipsômetro Vertex.

A projeção da copa foi determinada por meio da medição de oito raios com ângulos variáveis, metodologia recomendada para árvores com copas assimétricas (HUBER; RÖHLE, 1986; RÖHLE, 1986; SPIECKER, 1991 citados por NUTTO, 2001). Os raios da copa foram medidos com o auxílio de uma fita métrica, um hipsômetro Vertex e uma bússola.

Em seguida, os cálculos geométricos foram feitos com os pontos das coordenadas, determinados pela distância e o azimute da árvore. Com base nos pontos obtidos projetaram-se os polígonos e procedeu-se ao cálculo da área da copa, por meio do método triangular (RÖHLE, 1969 citado por NUTTO, 2001).

\section{Deformação residual longitudinal (DRL)}

A deformação residual longitudinal (DRL) foi tomada a 1,30 $\mathrm{m}$ do solo. As medidas foram realizadas ao redor do tronco nas orientações cardeais norte $\left(0^{\circ}-360^{\circ}\right)$, sul $\left(180^{\circ}\right)$, leste $\left(90^{\circ}\right)$ e oeste $\left(270^{\circ}\right)$ (figura 2).

Com intuito de avaliar a relação entre a DRL e as variáveis de crescimento das árvores, bem com as propriedades físicas e mecânicas da madeira, realizou-se uma análise de correlação simples de Pearson. Também para melhor compreensão da relação entre DRL e as variáveis de crescimento, estas foram estratificadas para cada espécie, em função da idade e avaliou-se a correlação, já que as árvores são relativamente jovens.
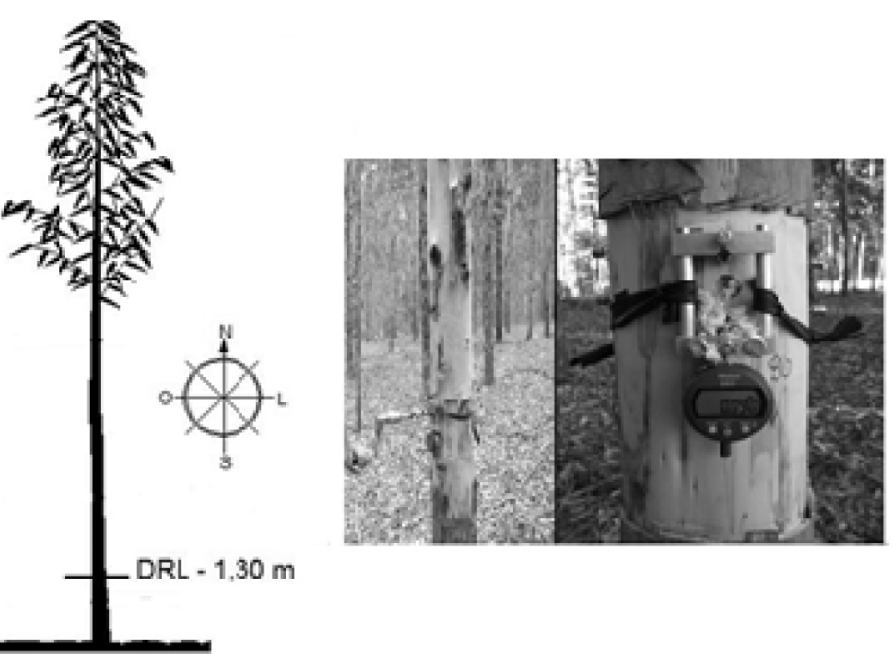

Figura 1. Posições e medição da deformação residual longitudinal.

Figure 1. Positions and measurement of residual longitudinal strain.

As medições foram realizadas de acordo com o método não destrutivo do "Centre de Cooperation Internationale en Recherche Agronomique Pour le Développement-Département Forêt" - CIRAD - Forêt ("Growth Strain Gauge" - Medidor de Deformação de Crescimento).

Utilizou-se um aparelho denominado extensômetro, no qual mede a deformação residual longitudinal (DRL) das fibras da madeira liberadas pela tensão de crescimento. Ao aparelho foram conectados dois pinos, com uma distância entre eles de $45 \mathrm{~mm}$ na direção da grã, fixados no tronco da árvore na altura das medidas, em uma região do tronco sem casca, a qual foi retirada para a mensuração da DRL. Posteriormente ao ajuste do aparelho, foi feito um furo entre os pinos de 2 $\mathrm{cm}$ de diâmetro e aproximadamente $30 \mathrm{~mm}$ de profundidade, com o uso de um arco de pua, a fim de liberar as tensões refletidas da contração longitudinal das fibras. Ao liberar a tensão, o valor em milímetros da DRL foi registrado diretamente no visor do relógio digital.

\section{Propriedades da madeira}

Para a determinação das propriedades físicas e mecânicas da madeira foram confeccionados corpos de prova retirados das toras obtidas na posição na altura de 1,30 m no solo, das árvores nas quais foram tomadas as DRLs (Figura 2). 
Após a confecção dos corpos de prova, os mesmos foram mensurados e ensaiados de acordo com as normas da Comissão Panamericana de Normas Técnicas (COPANT, 1972a), estabelecidas para a determinação da massa específica (COPANT 1972b), retratibilidade da madeira (COPANT 1972c) e a resistência à compressão paralela as fibras (adaptada da COPANT 464 (1972) (Figura 2).
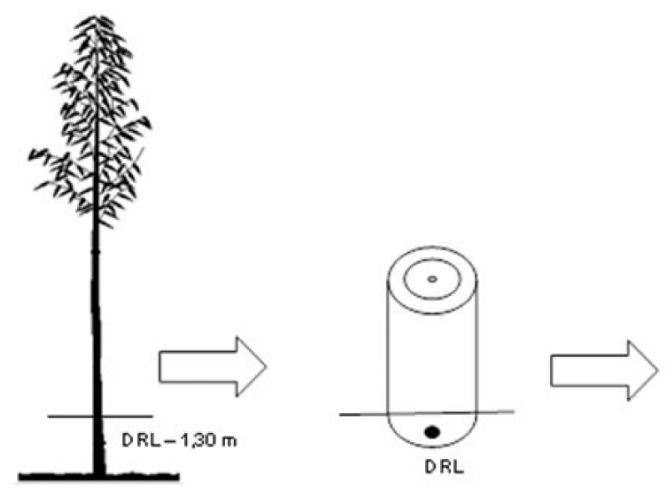

Propriedades fisicas e mecânica da madeira

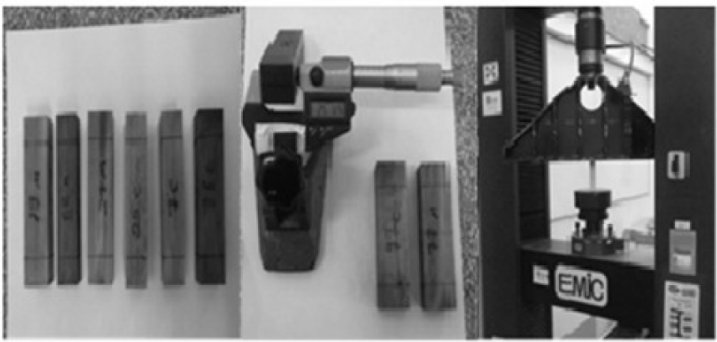

Figura 2. Amostragem para avaliação das propriedades físicas e mecânicas da madeira.

Figure 2. Sampling for evaluation of physical and mechanical properties of wood.

\section{Análise dos dados}

Com intuito de avaliar a relação entre a DRL e as variáveis de crescimento das árvores, bem com as propriedades físicas e mecânicas da madeira, realizou-se uma análise de correlação simples de Pearson. Também para melhor compreensão da relação entre DRL e as variáveis de crescimento, estas foram estratificadas para cada espécie, em função da idade e avaliou-se a correlação, já que as árvores são relativamente jovens.

\section{RESULTADOS E DISCUSSÃO}

\section{Correlação da DRL com as variáveis de crescimento da árvore}

Observa-se (Tabela 2) uma amplitude de variação de baixa a mediana, para as variáveis diâmetro à altura do peito (DAP), área da copa (Ac) e diâmetro da copa (Dc). A variável altura total da árvore (Ht) apresentou uma amplitude de variação considerada alta, mas em geral, as espécies avaliadas apresentaram um comportamento semelhante.

Os menores valores de DRL para as três espécies foram encontrados nas árvores de menor idade, aumentando conforme a idade. Ao comparar o presente trabalho com a literatura, quando foram utilizadas as mesmas espécies, o comportamento da DRL também foi semelhante, Trugilho et al. (2004) encontraram para madeira de E. dunnii, aos 8, 13, 15 e 19 anos de idade, implantados por mudas de sementes, valores médios para a DRL de 0,107, 0,113, 0111, 0,123 e 0,114 mm, respectivamente. Beltrame (2012) encontraram, para clones de E. grandis valores de 0,102, 0,156 e 0,094 mm, e para o E. saligna os valores de 0,093 e 0,108 mm.

Para a geração de produtos sólidos de madeira, busca-se espécies que apresentem os menores níveis da DRL, reduzindo os defeitos provenientes das tensões como empenamentos e rachaduras, consequentemente, eleva o aproveitamento e rendimento da madeira serrada.

Os maiores valores da DRL ocorreram nas árvores com maiores valores das variáveis de crescimento (DAP, Ht, Ac e Dc), ou seja, maiores taxas de crescimento proporcionaram maior desenvolvimento das tensões de crescimento. Essas taxas, no entanto, podem ser alteradas em função da qualidade do sítio, das práticas silviculturais e de manejo adotadas, promovendo a redução da tensão de crescimento e, consequentemente, maior equilíbrio e estabilidade das árvores, com alta produtividade para a geração de produtos sólidos.

Em relação às variáveis de crescimento, a espécie E. saligna, com apenas 52 meses de idade, apresentou valores médios semelhantes $\mathrm{e}$, em alguns casos superiores, quando comparada às espécies E. dunnii e E. grandis com idade de 65 meses, o que significa que houve maior incremento anual.

A influência das características de crescimento sobre a DRL vai depender da espécie e da idade, apresentando um comportamento diferenciado. Quando relacionada às variáveis de crescimento a DRL (Tabela 3), estas apresentaram, baixas correlações. A variável Ht foi a que proporcionou corre- 
lações significativas e positivas em todas as espécies, sendo que o E. dunnii e o E. saligna, apresentaram as maiores correlações, 0,65 e 0,75, respectivamente. O DAP também apresentou uma baixa correlação significativa com a DRL, para E. dunnii.

Tabela 2. Valores médios da deformação residual longitudinal e das variáveis de crescimento para as espécies $E$. dunnii, E. grandis e o E. saligna em diferentes idades.

Table 2. Mean values of longitudinal residual strain and growth variables for E. dunnii, E. grandis and E. saligna at different ages.

\begin{tabular}{|c|c|c|c|c|c|c|}
\hline \multirow{2}{*}{ Espécies } & \multirow{2}{*}{ Idade (meses) } & \multirow{2}{*}{ DRL } & \multicolumn{4}{|c|}{ Variáveis de crescimento } \\
\hline & & & DAP (cm) & $\mathrm{Ht}(\mathrm{m})$ & Ac (cm) & $\mathrm{Dc}(\mathrm{cm})$ \\
\hline \multirow{4}{*}{ E. dunnii } & 28 & $* *$ & $\begin{array}{c}10,48 \\
(21,45)^{\star}\end{array}$ & $\begin{array}{c}8,56 \\
(16,45)\end{array}$ & $\begin{array}{c}2,46 \\
(35,10)\end{array}$ & $\begin{array}{c}1,74 \\
(18,35) \\
\end{array}$ \\
\hline & 40 & $\begin{array}{c}0,086 \\
(25,93) \\
\end{array}$ & $\begin{array}{c}13,92 \\
(25,11) \\
\end{array}$ & $\begin{array}{c}10,80 \\
(25,11) \\
\end{array}$ & $\begin{array}{c}5,20 \\
(45,66) \\
\end{array}$ & $\begin{array}{c}2,51 \\
(23,39) \\
\end{array}$ \\
\hline & 52 & $\begin{array}{c}0,116 \\
(16,67) \\
\end{array}$ & $\begin{array}{c}17,12 \\
(14,89) \\
\end{array}$ & $\begin{array}{c}16,17 \\
(10,26) \\
\end{array}$ & $\begin{array}{c}6,52 \\
(33,31) \\
\end{array}$ & $\begin{array}{c}2,84 \\
(17,53) \\
\end{array}$ \\
\hline & 65 & $\begin{array}{c}0,126 \\
(22,72) \\
\end{array}$ & $\begin{array}{c}19,92 \\
(15,66) \\
\end{array}$ & $\begin{array}{c}18,52 \\
(14,39) \\
\end{array}$ & $\begin{array}{c}8,76 \\
(37,43) \\
\end{array}$ & $\begin{array}{c}3,28 \\
(18,84) \\
\end{array}$ \\
\hline \multirow{4}{*}{ E. grandis } & 28 & $* *$ & $\begin{array}{c}10,45 \\
(15,77) \\
\end{array}$ & $\begin{array}{c}8,63 \\
(12,08)\end{array}$ & $\begin{array}{c}2,69 \\
(34,00) \\
\end{array}$ & $\begin{array}{c}1,83 \\
(18,17) \\
\end{array}$ \\
\hline & 40 & $\begin{array}{c}0,085 \\
(31,38) \\
\end{array}$ & $\begin{array}{c}14,83 \\
(19,28) \\
\end{array}$ & $\begin{array}{c}11,03 \\
(15,80)\end{array}$ & $\begin{array}{c}5,49 \\
(39,67) \\
\end{array}$ & $\begin{array}{c}2,60 \\
(19,47) \\
\end{array}$ \\
\hline & 52 & $\begin{array}{c}0,095 \\
(20,88) \\
\end{array}$ & $\begin{array}{c}17,33 \\
(12,70) \\
\end{array}$ & $\begin{array}{c}16,29 \\
(10,29)\end{array}$ & $\begin{array}{c}10,45 \\
(30,07) \\
\end{array}$ & $\begin{array}{c}3,61 \\
(15,37) \\
\end{array}$ \\
\hline & 65 & $\begin{array}{c}0,093 \\
(14,02)\end{array}$ & $\begin{array}{c}19,45 \\
(15,17) \\
\end{array}$ & $\begin{array}{c}19,42 \\
(8,51) \\
\end{array}$ & $\begin{array}{c}10,62 \\
(44,10) \\
\end{array}$ & $\begin{array}{c}3,58 \\
(23,59) \\
\end{array}$ \\
\hline \multirow{3}{*}{ E. saligna } & 28 & $\begin{array}{c}0,072 \\
(11,77)\end{array}$ & $\begin{array}{l}10,30 \\
(8,21)\end{array}$ & $\begin{array}{c}9,30 \\
(7,21)\end{array}$ & $\begin{array}{c}2,41 \\
(25,78)\end{array}$ & $\begin{array}{c}1,74 \\
(13,20)\end{array}$ \\
\hline & 40 & $\begin{array}{c}0,082 \\
(18,50) \\
\end{array}$ & $\begin{array}{c}13,92 \\
(18,00) \\
\end{array}$ & $\begin{array}{c}11,83 \\
(20,03) \\
\end{array}$ & $\begin{array}{c}5,30 \\
(27,47) \\
\end{array}$ & $\begin{array}{c}2,57 \\
(14,55) \\
\end{array}$ \\
\hline & 52 & $\begin{array}{c}0,111 \\
(12,81)\end{array}$ & $\begin{array}{c}19,55 \\
(12,93)\end{array}$ & $\begin{array}{l}17,95 \\
(9,43)\end{array}$ & $\begin{array}{c}12,21 \\
(29,06)\end{array}$ & $\begin{array}{c}3,89 \\
(14,93)\end{array}$ \\
\hline
\end{tabular}

DRL - Deformação residual longitudinal; DAP - Diâmetro na altura do peito; Ht - Altura total; Ac - Área da copa; Dc - Diâmetro da copa. * Valores entre parênteses referem-se ao coeficiente de variação (\%). ** Não foram mensurados a DRL para a espécies de $E$. dunnii e $E$. grandis aos 28 meses de idade

Tabela 3. Correlação geral entre as variáveis de crescimento e a deformação residual longitudinal para as espécies E. dunnii, E. grandis e o E. saligna.

Table 3. General correlation matrix between the growth variables and the longitudinal residual strain for the species $E$. dunnii, E. grandis and E. saligna.

\begin{tabular}{llcccc}
\hline Espécies & & DRL $(\mathbf{m m})$ & DAP $(\mathbf{c m})$ & Ht $(\mathbf{m})$ & Dc $(\mathbf{c m})$ \\
\hline E. dunnii & DRL & 1,00 & $0,36^{*}$ & $0,65^{\star *}$ & 0,05 \\
E. grandis & DRL & 1,00 & 0,29 & $0,41^{*}$ & 0,10 \\
E. saligna & DRL & 1,00 & $0,77^{\star *}$ & $0,75^{\star *}$ & $0,75^{\star *}$ \\
\hline
\end{tabular}

DRL - Deformação residual longitudinal; DAP - Diâmetro na altura do peito; $\mathrm{Ht}$ - Altura total; Dc - Diâmetro da copa. ** e * significativo a I e $5 \%$ de probabilidade, respectivamente.

Em E. saligna, a DRL apresentou-se uma alta correlação positiva e significativa com todas as variáveis de crescimento. Diante disto, fica evidente que as variáveis de crescimento tendem a influenciar na DRL, principalmente para essa espécie, corroborando com Lima et al. (2004), ao afirmarem que a DRL se correlaciona com as variáveis de crescimento de fácil medição.

Para melhor compreensão da relação entre DRL e as variáveis de crescimento, estas foram estratificadas para cada espécie, em função da idade (Tabela 4).

Para E. dunnii, os valores obtidos da correlação da DRL com as variáveis de crescimento foram baixos, para a idade de 40 meses, indicando uma fraca influência dessas variáveis na DRL. Para a idade de 52 meses, as correlações foram moderadas e negativas, com exceção da altura total, que foi positiva, porém sem correlação significativa. Aos 65 meses de idade, apenas a variável diâmetro da copa apresentou uma alta correlação com a DRL, indicando maior influência dessa variável na DRL.

Em E. grandis, com 40 e 65 meses, as correlações foram baixas, não havendo relação dessas variáveis com a DRL (Tabela 3). Da mesma forma, verificou-se essa mesma tendência para o E. saligna nas idades de 28, 40 e 52 meses. 
Braz et al. - Relação entre a deformação residual longitudinal com

crescimento e propriedades da madeira de espécies de Eucalyptus

Tabela 4. Correlação com as idades estratificadas das variáveis de crescimento com a deformação residual longitudinal para o E. dunnii, E. grandis e o E. saligna.

Table 4. Correlation with ages stratified for growth variables with longitudinal residual strain for E. dunnii, E. grandis and E. saligna.

\begin{tabular}{|c|c|c|c|c|}
\hline \multicolumn{5}{|c|}{ Eucalyptus dunnii } \\
\hline Idade (meses) & & DAP (cm) & $\mathrm{Ht}(\mathrm{m})$ & $\mathrm{Dc}(\mathrm{cm})$ \\
\hline 40 & $\mathrm{DRL}(\mathrm{mm})$ & 0,17 & 0,18 & 0,21 \\
\hline 52 & $\mathrm{DRL}(\mathrm{mm})$ & $-0,60$ & 0,69 & $-0,56$ \\
\hline 65 & $\mathrm{DRL}(\mathrm{mm})$ & $-0,42$ & 0,22 & $-0,75^{\star *}$ \\
\hline \multicolumn{5}{|c|}{ Eucalyptus grandis } \\
\hline Idade (meses) & & DAP (cm) & $\mathrm{Ht}(\mathrm{m})$ & $\mathrm{Dc}(\mathrm{cm})$ \\
\hline 40 & $\mathrm{DRL}(\mathrm{mm})$ & $-0,15$ & 0,14 & $-0,10$ \\
\hline 52 & $\mathrm{DRL}(\mathrm{mm})$ & 0,56 & 0,75 & 0,06 \\
\hline 65 & $\mathrm{DRL}(\mathrm{mm})$ & 0,08 & $-0,04$ & $-0,30$ \\
\hline \multicolumn{5}{|c|}{ Eucalyptus saligna } \\
\hline Idade (meses) & & DAP (cm) & $\mathrm{Ht}(\mathrm{m})$ & $\mathrm{Dc}(\mathrm{cm})$ \\
\hline 28 & $\mathrm{DRL}(\mathrm{mm})$ & 0,24 & 0,12 & 0,13 \\
\hline 40 & $\mathrm{DRL}(\mathrm{mm})$ & 0,42 & 0,30 & 0,33 \\
\hline 52 & $\mathrm{DRL}(\mathrm{mm})$ & 0,12 & $-0,01$ & 0,06 \\
\hline
\end{tabular}

DRL - Deformação residual longitudinal; DAP - Diâmetro na altura do peito; Ht - Altura total; Dc - Diâmetro da copa. ** e * significativo a I e $5 \%$ de probabilidade, respectivamente.

Ao avaliar a relação das variáveis de crescimento com a DRL dentro de cada idade, separadamente para as espécies utilizadas, em geral, observaram-se correlações fracas. Contudo, as variáveis tenderam a uma alta correlação, de maior magnitude em E. dunnii e E. grandis com 52 meses.

Os trabalhos citados na literatura são bastante controversos quanto às correlações das características de crescimento das árvores com a DRL. Muneri et al. (1999), encontraram correlações positivas da DRL, com a altura $(0,44)$ e diâmetro das árvores $(0,51)$ para o E. cloeziana.

Wilkins e Kitahara (1991), ao estudarem o E. grandis, com 12,5 e 22 anos, encontraram uma correlação negativa entre DRL e DAP $(r=-0,70)$. Segundo Yang et al. (2001), os valores de DRL tendem a diminuir com a altura, em árvores jovens de E. globulus.

Os resultados obtidos no presente trabalho, no entanto, estão em conformidade com os encontrados com os trabalhos citados na literatura. Fernandes (1982), Souza (2002) e Trugilho et al. (2004) não encontraram correlações significativas entre a DRL e as características de crescimento para o E. dunnii, em diferentes idades. Gonçalves (2007) não encontrou tendência quando as classes de altura se relacionaram com a DRL, possuindo correlação linear simples não significativa. Lima et al. (2004), também não encontraram correlação entre as características de crescimento e a DRL.

Miranda e Nahuz (1999) observaram variação da DRL, em clones de E. saligna, com nove anos de idade, sem correlação significativa com as características de crescimento da árvore.

A falta de correlação pode ser justificada em função de a DRL ser uma medida, tomada indiretamente, para determinação das tensões de crescimento, (TRUGILHO et al., 2006). Outra situação são as diferentes características das variáveis de crescimento de uma árvore para outra ou entre as espécies, o que pode interferir na interação com a DRL, além de outros fatores, como os ambientais e silviculturais.

As variáveis de crescimento são fundamentais para avaliar o desenvolvimento dos plantios florestais, e influenciam, também, nas propriedades e na qualidade da madeira, podendo ser manejadas, o que pode auxiliar na redução dos níveis das tensões de crescimento, haja visto a relação entre algumas variáveis e a DRL.

O espaçamento e desbaste, por exemplo, permitem direcionar as copas das árvores, o que pode influenciar nas tensões de crescimento, segundo Lima et al. (2000) enquanto as intensidades dos desbastes não provocarem uma reorientação das copas, eles poderão reduzir o nível das tensões. Dessa forma, espera-se que o E. saligna tende a reduzir as tensões de crescimento, em função do diâmetro da copa, uma vez que apresentaram uma forte correlação.

Nota-se pelos resultados obtidos, a influência das variáveis de crescimento na DRL vai depender da idade e da espécie, apresentando comportamentos diferenciados. Observa-se, por exemplo, a relação da DRL com a variável DAP, ora é diretamente proporcional, ora, inversamente, o que evidencia que a DRL é uma variável altamente dinâmica e que pode ser influenciada por diversos fatores. 


\section{Correlação da DRL com as propriedades físicas da madeira}

Consta na Tabela 5, os valores médios de algumas propriedades físicas da madeira, em função da idade, para E. dunnii, E. grandis e E. saligna.

Tabela 5. Valores médios de propriedades físicas em função das idades para as espécies E. dunnii, E. grandis e E. saligna.

Table 5. Mean values of the physical properties for ages for the species E. dunnii, E. grandis and E. saligna.

\begin{tabular}{|c|c|c|c|c|c|c|c|c|}
\hline Espécies & Idade (meses) & MEA $\left(\mathrm{kg} \cdot \mathrm{m}^{-3}\right)$ & MEB $\left(\mathrm{kg} \cdot \mathrm{m}^{-3}\right)$ & CT (\%) & CR (\%) & CL (\%) & CV (\%) & $\mathrm{CA}$ \\
\hline \multirow{3}{*}{ E. dunnii } & 40 & $\begin{array}{c}565 \\
(10,04)^{*} \\
\end{array}$ & $\begin{array}{c}433 \\
(8,47) \\
\end{array}$ & $\begin{array}{c}9,27 \\
(23,93) \\
\end{array}$ & $\begin{array}{c}4,40 \\
(18,75) \\
\end{array}$ & $\begin{array}{c}0,40 \\
(38,46) \\
\end{array}$ & $\begin{array}{c}13,59 \\
(19,27) \\
\end{array}$ & $\begin{array}{c}2,15 \\
(19,63) \\
\end{array}$ \\
\hline & 52 & $\begin{array}{c}603 \\
(9,46)\end{array}$ & $\begin{array}{c}431 \\
(12,40)\end{array}$ & $\begin{array}{c}10,61 \\
(23,14)\end{array}$ & $\begin{array}{c}4,93 \\
(22,09)\end{array}$ & $\begin{array}{c}0,36 \\
(23,64)\end{array}$ & $\begin{array}{c}15,31 \\
(20,54)\end{array}$ & $\begin{array}{c}2,22 \\
(15,76)\end{array}$ \\
\hline & 65 & $\begin{array}{c}635 \\
(9,60) \\
\end{array}$ & $\begin{array}{c}477 \\
(7,74) \\
\end{array}$ & $\begin{array}{c}10,82 \\
(12,36) \\
\end{array}$ & $\begin{array}{c}5,50 \\
(17,34) \\
\end{array}$ & $\begin{array}{c}0,37 \\
(22,50)\end{array}$ & $\begin{array}{c}16,03 \\
(10,79) \\
\end{array}$ & $\begin{array}{c}2,02 \\
(17,29) \\
\end{array}$ \\
\hline Média & & $\begin{array}{c}615 \\
(10,52)\end{array}$ & $\begin{array}{c}460 \\
(9,92)\end{array}$ & $\begin{array}{c}10,43 \\
(17,44)\end{array}$ & $\begin{array}{c}5,15 \\
(19,92)\end{array}$ & $\begin{array}{c}0,38 \\
(26,40)\end{array}$ & $\begin{array}{c}15,35 \\
(15,37)\end{array}$ & $\begin{array}{c}2,08 \\
(17,30)\end{array}$ \\
\hline \multirow{3}{*}{ E. grandis } & 40 & $\begin{array}{c}474 \\
(8,15) \\
\end{array}$ & $\begin{array}{c}385 \\
(7,04) \\
\end{array}$ & $\begin{array}{c}7,28 \\
(7,80) \\
\end{array}$ & $\begin{array}{c}3,81 \\
(12,86) \\
\end{array}$ & $\begin{array}{c}0,41 \\
(35,55)\end{array}$ & $\begin{array}{c}11,18 \\
(7,17) \\
\end{array}$ & $\begin{array}{c}1,99 \\
(14,73) \\
\end{array}$ \\
\hline & 52 & $\begin{array}{c}510 \\
(6,76)\end{array}$ & $\begin{array}{c}417 \\
(5,61)\end{array}$ & $\begin{array}{c}7,58 \\
(7,57)\end{array}$ & $\begin{array}{c}4,14 \\
(19,02)\end{array}$ & $\begin{array}{c}0,36 \\
(19,95)\end{array}$ & $\begin{array}{l}11,72 \\
(7,97)\end{array}$ & $\begin{array}{c}1,88 \\
(17,86)\end{array}$ \\
\hline & 65 & $\begin{array}{c}499 \\
(10,94)\end{array}$ & $\begin{array}{c}403 \\
(7,69)\end{array}$ & $\begin{array}{c}7,72 \\
(13,12)\end{array}$ & $\begin{array}{c}4,10 \\
(11,13)\end{array}$ & $\begin{array}{c}0,35 \\
(27,52)\end{array}$ & $\begin{array}{c}11,73 \\
(10,62)\end{array}$ & $\begin{array}{c}1,91 \\
(12,20)\end{array}$ \\
\hline Média & & $\begin{array}{c}496 \\
(10,02)\end{array}$ & $\begin{array}{c}402 \\
(7,62)\end{array}$ & $\begin{array}{c}7,61 \\
(11,51)\end{array}$ & $\begin{array}{c}4,05 \\
(12,68)\end{array}$ & $\begin{array}{c}0,36 \\
(28,49)\end{array}$ & $\begin{array}{l}11,62 \\
(9,58)\end{array}$ & $\begin{array}{c}1,92 \\
(13,16)\end{array}$ \\
\hline \multirow{2}{*}{ E. saligna } & 40 & $\begin{array}{c}533 \\
(6,52) \\
\end{array}$ & $\begin{array}{c}433 \\
(7,27) \\
\end{array}$ & $\begin{array}{c}8,38 \\
(5,39) \\
\end{array}$ & $\begin{array}{c}4,17 \\
(7,00) \\
\end{array}$ & $\begin{array}{c}0,39 \\
(25,65)\end{array}$ & $\begin{array}{l}12,54 \\
(4,64) \\
\end{array}$ & $\begin{array}{c}2,03 \\
(7,07) \\
\end{array}$ \\
\hline & 52 & $\begin{array}{c}583 \\
(9,47)\end{array}$ & $\begin{array}{c}459 \\
(6,78)\end{array}$ & $\begin{array}{c}8,01 \\
(3,96)\end{array}$ & $\begin{array}{c}4,17 \\
(13,60)\end{array}$ & $\begin{array}{c}0,40 \\
(2,93)\end{array}$ & $\begin{array}{l}12,20 \\
(5,39)\end{array}$ & $\begin{array}{c}1,97 \\
(9,62)\end{array}$ \\
\hline Média & & $\begin{array}{c}553 \\
(9,07)\end{array}$ & $\begin{array}{c}444 \\
(7,58)\end{array}$ & $\begin{array}{c}8,22 \\
(5,21)\end{array}$ & $\begin{array}{c}4,17 \\
(9,69)\end{array}$ & $\begin{array}{c}0,40 \\
(18,86)\end{array}$ & $\begin{array}{l}12,40 \\
(4,92)\end{array}$ & $\begin{array}{c}2,00 \\
(7,93)\end{array}$ \\
\hline Média geral & & $\begin{array}{c}556 \\
(14,01)\end{array}$ & $\begin{array}{c}433 \\
(10,68)\end{array}$ & $\begin{array}{c}8,92 \\
(20,81)\end{array}$ & $\begin{array}{c}4,54 \\
(20,21)\end{array}$ & $\begin{array}{c}0,37 \\
(25,68)\end{array}$ & $\begin{array}{c}13,34 \\
(18,22)\end{array}$ & $\begin{array}{c}2,01 \\
(14,83) \\
\end{array}$ \\
\hline
\end{tabular}

MEA - Massa específica aparente; MEB - Massa específica básica; CT - Contração tangencial; CR - Contração radial (\%); CL - Contração longitudinal; CV - Contração volumétrica e CA - Coeficiente de anisotropia. * valores entre parênteses referem-se ao coeficiente de variação (\%).

Observa-se, em geral, baixa correlação entre a DRL e as propriedades físicas avaliadas (Tabela 6), principalmente para E. dunnii e E. grandis. Exceto a MEA e MEB que apresentaram alta correlação com a DRL para o E. saligna.

Tabela 6. Correlação geral entre a deformação residual longitudinal e as propriedades físicas para as espécies de E. dunnii, E. grandis e E. saligna.

Table 6. General correlation between the longitudinal residual strain and physical properties of E. dunnii, E. grandis and E. saligna.

\begin{tabular}{lccccccccc}
\hline Espécies & & DRL $(\mathbf{m m})$ & MEA (kg.m $\left.{ }^{-3}\right)$ & MEB (kg.m $\left.\mathbf{m}^{-3}\right)$ & CT (\%) & CR (\%) & CL (\%) & CV (\%) & CA \\
\hline E. dunnii & DRL & 1,00 & $0,51^{* *}$ & $0,41^{*}$ & 0,19 & $0,43^{*}$ & $-0,32$ & 0,30 & $-0,27$ \\
E. grandis & DRL & 1,00 & 0,23 & 0,23 & 0,08 & 0,07 & $-0,09$ & 0,09 & $-0,07$ \\
E. saligna & DRL & 1,00 & $0,77^{* *}$ & $0,80^{* *}$ & $-0,12$ & 0,27 & 0,02 & 0,08 & $-0,28$ \\
\hline
\end{tabular}

DRL - Deformação residual longitudinal; MEA - Massa específica aparente; MEB - Massa específica básica; CT - Contração tangencial; CR - Contração radial (\%); CL - Contração longitudinal; CV - Contração volumétrica e AC - Anisotropia de contração. ** e * significativo a I e 5\% de probabilidade, respectivamente.

Segundo Malan (1984), a redução dos níveis de tensão de crescimento reduz a massa específica básica, informação que pode ser empregada na seleção de clones. Verificou-se no presente trabalho, que a madeira com maior massa específica, tanto aparente quanto a básica (E. dunnii, 65 meses de idade) apresentou a maior valor médio para a DRL.

Para avaliar o efeito da idade, estas foram estratificadas e avaliou-se a correlação da DRL com as propriedades físicas para as espécies de E. dunnii, E. grandis e E. saligna. Em geral, a madeira de E. dunnii, aos 40 e 65 meses de idade, apresentaram baixa correlação entre a DRL e as propriedades físicas da madeira (Tabela 7). 
Braz et al. - Relação entre a deformação residual longitudinal com

crescimento e propriedades da madeira de espécies de Eucalyptus

Tabela 7. Correlação com as idades estratificadas entre a deformação residual longitudinal e as propriedades físicas para a espécie de E. dunnii, E. grandis e E. saligna.

Table 7. Correlation with ages stratified residual longitudinal strain and physical properties of the species $E$. dunnii, E. grandis and E. saligna.

\begin{tabular}{|c|c|c|c|c|c|c|c|c|}
\hline \multicolumn{9}{|c|}{ Eucalyptus dunnii } \\
\hline Idade (meses) & & MEA $\left(\mathrm{kg} \cdot \mathrm{m}^{-3}\right)$ & MEB $\left(\mathrm{kg} \cdot \mathrm{m}^{-3}\right)$ & CT (\%) & CR (\%) & CL (\%) & CV (\%) & CA \\
\hline 40 & $\mathrm{DRL}(\mathrm{mm})$ & 0,35 & 0,19 & 0,32 & 0,77 & $-0,13$ & 0,48 & $-0,35$ \\
\hline 52 & $\mathrm{DRL}(\mathrm{mm})$ & $-0,51$ & $-0,17$ & $-0,90^{*}$ & $-0,89^{*}$ & $-0,62$ & $-0,97^{\star *}$ & $-0,24$ \\
\hline 65 & $\mathrm{DRL}(\mathrm{mm})$ & 0,22 & 0,09 & $-0,18$ & 0,14 & $-0,50^{*}$ & $-0,08$ & $-0,21$ \\
\hline \multicolumn{9}{|c|}{ Eucalyptus grandis } \\
\hline Idade (meses) & & $\operatorname{MEA}\left(\mathrm{kg} \cdot \mathrm{m}^{-3}\right)$ & MEB $\left(\mathrm{kg} \cdot \mathrm{m}^{-3}\right)$ & CT (\%) & CR (\%) & CL (\%) & CV (\%) & CA \\
\hline 40 & $\mathrm{DRL}(\mathrm{mm})$ & $-0,43$ & $-0,47$ & $-0,84$ & 0,09 & $-0,52$ & $-0,61$ & $-0,60$ \\
\hline 52 & $\mathrm{DRL}(\mathrm{mm})$ & 0,87 & 0,78 & $-0,83$ & $-0,52$ & 0,33 & $-0,87$ & 0,07 \\
\hline 65 & $\mathrm{DRL}(\mathrm{mm})$ & 0,12 & 0,13 & 0,16 & 0,07 & 0,29 & 0,21 & 0,18 \\
\hline \multicolumn{9}{|c|}{ Eucalyptus saligna } \\
\hline Idade (meses) & & MEA $\left(\mathrm{kg} \cdot \mathrm{m}^{-3}\right)$ & MEB $\left(\mathrm{kg} \cdot \mathrm{m}^{-3}\right)$ & CT (\%) & CR (\%) & CL (\%) & CV (\%) & CA \\
\hline 40 & $\mathrm{DRL}(\mathrm{mm})$ & $-0,44$ & 0,25 & 0,65 & 0,34 & $-0,25$ & 0,60 & 0,16 \\
\hline 52 & $\mathrm{DRL}(\mathrm{mm})$ & $-0,48$ & $-0,42$ & 0,77 & 0,69 & 0,46 & $0,90^{*}$ & $-0,53$ \\
\hline
\end{tabular}

DRL - Deformação residual longitudinal; MEA - Massa específica aparente; MEB - Massa específica básica; CT - Contração tangencial; CR - Contração radial (\%); CL - Contração longitudinal; CV - Contração volumétrica e AC - Anisotropia de contração. ** e * significativo a I e $5 \%$ de probabilidade, respectivamente.

Verificou-se para o E. dunni e E. grandis aos 52 meses, que as variáveis CT, CR e CV apresentaram uma alta correlação negativa com DRL. O E. saligna na idade de 40 meses, apresentou uma correlação moderada entre a DRL com a CT e CV, para as demais variáveis as correlações foram baixas. Para a idade de 52 meses, a DRL apresentou correlações também moderadas para a CT, CR e AC.

Na literatura, observou-se baixos valores de correlação entre a DRL e algumas propriedades físicas da madeira. Lima et al. (2004), ao correlacionarem a DRL e algumas propriedades da madeira de clones de E. urophylla x E. grandis, relataram uma correlação significativa entre a DRL e a densidade básica.

Gonçalves et al. (2010) verificaram para híbridos de E. urophylla $\times$ E. grandis uma correlação baixa da DRL, com a densidade básica $(0,32)$, contração radial $(0,38)$, contração tangencial $(0,56)$ e contração volumétrica $(0,39)$, sendo significativa, apenas para a contração tangencial. Os mesmos autores encontraram, também, uma baixa correlação da DRL com o fator anisotrópico $(-0,17)$.

Trugilho et al. (2006) ao estudarem híbridos naturais do gênero Eucalyptus aos seis anos, relataram uma correlação da DRL positiva e significativa com a densidade básica $(0,52)$, contração volumétrica $(0,69)$, radial $(0,46)$ e tangencial $(0,71)$, já para o coeficiente de anisotropia não encontraram correlação.

Visto os resultados obtidos no presente estudo, bem como os trabalhos citados, observa-se também, uma grande variabilidade dos valores obtidos das correlações entre a DRL e propriedades físicas da madeira. Verificou-se, no entanto, que os valores obtidos das correlações apresentaram uma maior magnitude de influência das retrações da madeira na DRL, indicando que a DRL pode afetar as características físicas. Dessa forma, os resultados indicam que os níveis da DRL podem ser estimados em função das retrações dimensionais da madeira.

Isso pode ser justificado pelo fato da DRL e as retrações na madeira estarem relacionadas diretamente ao comportamento dimensional e aos defeitos na madeira, como o empenamento, fendilhamento e rachaduras; a perda de água também promove alterações na estrutura do tecido lenhoso o que pode influenciar na medida dos valores da DRL.

De acordo com Delucis et al. (2014) a modificação na estrutura do tecido lenhoso, devido ao mecanismo de dessorção de água, provavelmente causa a alteração dos valores de DRL no sentido de quanto maior a intensidade da retração, menor o nível de tensão de crescimento, o efeito do esforço de retração ocorre no sentido contrário ao efeito do esforço de liberação da tensão de crescimento. No presente trabalho, observa-se que o comportamento foi inverso, uma vez que as maiores retrações foram obtidas na madeira que apresentou os maiores valores da DRL, no caso $o$ E. dunnii, confirmando o comportamento bastante disperso das deformações.

Verificou-se um comportamento bastante disperso da DRL quando relacionado as propriedades físicas da madeira, dependendo diretamente da idade e também da espécie. 


\section{Correlação da DRL com a Compressão paralela as fibras}

A média geral para o módulo de elasticidade (MOE - modulo de elasticidade a compressão paralela a grã ou rigidez) e módulo de ruptura (MOR - modulo de ruptura ou tensão máxima de ruptura a compressão paralela) foi de $4302 \mathrm{MPa}$ e 35,12 MPa, respectivamente. Os valores médios, obtidos para E. dunnii, E. grandis e E. saligna, foram 4693, 3904 e $4283 \mathrm{Mpa}$, para o MOE, e 37,74, 32,74 e 34,39 MPa para o MOR, respectivamente. Os valores médios para o MOE e MOR variaram de $3569 \mathrm{MPa}$ e 29,66 MPa (E. grandis aos 40 meses de idade) a $4942 \mathrm{MPa}$ e 39,80 MPa (E. dunnii, aos 65 meses de idade) (Tabela 8).

Tabela 8. Valores médios do módulo de elasticidade e módulo de ruptura da resistência a compressão paralela as fibras. Table 8. Mean values of the modulus of elasticity and modulus of rupture resistance to parallel to fiber compression.

\begin{tabular}{lccc}
\hline Espécie & Idade (meses) & $\begin{array}{c}\text { Resistência a Compressão paralela a fibras (MPa) } \\
\text { MOE }\end{array}$ & MOR \\
\hline \multirow{2}{*}{ E. dunnii } & 40 & $3971(12,98)^{*}$ & $32,17(13,05)$ \\
& 52 & $4620(4,85)$ & $36,73(5,97)$ \\
Média & 65 & $4942(8,40)$ & $39,80(8,76)$ \\
\hline \multirow{2}{*}{ E. grandis } & 40 & $4693(11,65)$ & $37,74(11,91)$ \\
& 52 & $3569(10,37)$ & $29,66(7,65)$ \\
\hline Média & 65 & $4109(6,96)$ & $34,04(7,09)$ \\
\hline \multirow{2}{*}{ E. saligna } & & $3959(4,43)$ & $33,37(3,98)$ \\
\hline Média & 40 & $3904(7,45)$ & $32,74(6,98)$ \\
Média geral & 52 & $4189(4,12)$ & $33,98(2,83)$ \\
\hline MOE -Món & $4415(2,21)$ & $34,96(3,32)$ \\
\hline
\end{tabular}

MOE - Módulo de elasticidade; MOR - Módulo de ruptura. * valores entre parênteses referem-se ao coeficiente de variação (\%)

Os coeficientes de variação tanto para o MOE quanto para o MOR foram baixos, evidenciando uma homogeneidade entre as amostras utilizadas, indicando haver menor variabilidade na madeira, o que a torna de melhor qualidade em relação a essas variáveis. Porém, de menor potencial de seleção genética.

A forte correlação foi observada entre a DRL com o MOE e MOR para o E. dunniii. Para as demais espécies, as correlações tenderam de moderada à relativamente baixas, indicando baixa relação entre a DRL e as variáveis analisadas (Tabela 9).

Tabela 9. Correlação geral entre a deformação residual longitudinal e a resistência compressão paralela as fibras e o módulo de elasticidade para as espécies de E. dunnii, E. grandis e E. saligna.

Table 9. General correlation between the longitudinal residual strain and parallel to fiber compression strength and the modulus of elasticity for the species E. dunnii, E. grandis and E. saligna.

\begin{tabular}{llccc}
\hline Espécie & & DRL $(\mathbf{m m})$ & MOE $(\mathbf{M P a})$ & MOR (MPa) \\
\hline E. dunnii & DRL & 1,00 & $0,67^{* *}$ & $0,68^{* *}$ \\
E. grandis & DRL & 1,00 & 0,36 & $0,48^{* *}$ \\
E. saligna & DRL & 1,00 & 0,52 & 0,45
\end{tabular}

MOE - Módulo de elasticidade; MOR - Módulo de ruptura. ** e * significativo a I e 5\% de probabilidade, respectivamente.

Ao avaliar o efeito da idade, fez-se a estratificação e avaliou-se a correlação da DRL com o MOE e MOR da resistência à compressão paralela a fibras para as espécies $\mathrm{O}$ E. dunnii aos 52 meses de idade, foi a que apresentou uma correlação forte entre a DRL e as variáveis MOE e MOR. Para as demais espécies e idades, em geral, a correlação entre a DRL e as propriedades avaliadas foram fracas (Tabela 10).

Na literatura, diversos trabalhos realizados não encontraram uma correlação entre a DRL e as propriedades mecânicas da madeira, entre elas a compressão paralela às fibras.

Lima et al. (2004) mencionaram não haver uma correlação entre a DRL e a compressão paralela às fibras, ao estudarem clones de eucalipto, sendo quatro híbridos naturais de E. grandis e um híbrido do E. urophylla x E. grandis. Trugilho et al. (2007), ao avaliar onze clones híbridos naturais de Eucalyptus, com seis anos de idade, não encontraram uma correlação da DRL com o MOE. 
Braz et al. - Relação entre a deformação residual longitudinal com

crescimento e propriedades da madeira de espécies de Eucalyptus

Tabela 10. Correlação com as idades estratificadas entre a deformação residual longitudinal e o módulo de elasticidade e ruptura para a espécie de E. dunnii, E. grandis e E. saligna.

Table 10. Correlation with the stratified ages between the longitudinal residual strain and the elasticity and rupture module to the species E. dunnii, E. grandis and E. saligna.

\begin{tabular}{lccc}
\hline Eucalyptus dunnii & & & \\
\hline Idade (meses) & & MOE (MPa) & MOR (MPa) \\
\hline 40 & $\mathrm{DRL}(\mathrm{mm})$ & 0,56 & 0,42 \\
52 & $\mathrm{DRL}(\mathrm{mm})$ & 0,84 & 0,81 \\
65 & $\mathrm{DRL}(\mathrm{mm})$ & 0,39 & 0,46 \\
\hline Eucalyptus grandis & & & \\
\hline Idade (meses) & & MOE (MPa) & MOR (MPa) \\
\hline 40 & $\mathrm{DRL}(\mathrm{mm})$ & 0,11 & 0,08 \\
52 & $\mathrm{DRL}(\mathrm{mm})$ & 0,40 & 0,53 \\
65 & $\mathrm{DRL}(\mathrm{mm})$ & 0,04 & 0,17 \\
\hline Eucalyptus saligna & & & \\
\hline Idade (meses) & & MOE (MPa) & MOR (MPa) \\
\hline 40 & $\mathrm{DRL}(\mathrm{mm})$ & 0,19 & 0,49 \\
52 & $\mathrm{DRL}(\mathrm{mm})$ & $-0,69$ & $-0,14$ \\
\hline
\end{tabular}

MOE - Módulo de elasticidade; MOR - Módulo de ruptura. ** e ${ }^{*}$ significativo a I e $5 \%$ de probabilidade, respectivamente.

Gonçalves et al. (2010), analisando clones dos híbridos de E. urophylla x E. grandis, encontraram uma correlação não significativa entre a DRL e a compressão paralela às fibras. Da mesma semelhante, Beltrame (2012) encontrou baixas correlações e não significativas para a madeira de Eucalyptus, entre a DRL e o MOE e a resistência à compressão paralela às fibras.

Em geral, observou-se um comportamento diferenciado quando as idades foram analisadas conjunta ou isoladamente, dentro de cada espécie. Para a primeira situação, verificou-se uma maior magnitude da relação da DRL com o MOE e MOR da resistência à compressão paralela às fibras. Quando as idades foram analisadas separadamente, dentro de cada espécie, não foi encontrada uma tendência da influência da DRL nos MOE e MOR, evidenciando uma correlação relativamente baixa.

O MOE e MOR encontrados na idade de 52 meses, foram os que melhores se correlacionaram com a DRL, em todas as espécies, indicando uma influência da DRL sobre as características de resistência mecânica para essa idade. Não se evidenciam qualquer influência da idade, visto que, para as outras, o comportamento foi diferente.

Programas de melhoramento genético florestal, associados aos incrementos de novas práticas silviculturais devem ser direcionados para a produção de madeira com características desejáveis para a obtenção da madeira sólida, com resistência mecânica, porém com baixa ocorrência das tensões de crescimento.

Em geral, os resultados obtidos devem-se principalmente ao fato de se ter trabalhado com arvores com $100 \%$ de lenho juvenil, cujas características, físicas, químicas, anatômicas e mecânicas são muito distintas daquelas de madeira adulta, conforme muito bem documentado na literatura.

O presente estudo com árvores jovens permite que esta pesquisa tenha continuidade em estudos futuros nos mesmos plantios em que se poderá avaliar se os resultados obtidos se confirmam em árvores adultas. Desta forma, poderá se confirmar ou não as tendências já observadas, com melhoria nas características e relações das propriedades estudadas com a DRL.

\section{CONCLUSÕES}

Com base nos resultados obtidos, concluiu-se que:

As correlações entre a DRL e as variáveis de crescimento das árvores, propriedades físicas e mecânicas apresentaram uma alta dispersão;

O E. saligna apresentou os melhores resultados de correlação entre a DRL e as variáveis de crescimento. Ao estratificar por idade, o E.dunni e E. grandis, ambos com 52 meses, foram os que apresentaram os melhores resultados;

As árvores na idade de 52 meses foram as que apresentaram os melhores resultados de correlação entre a DRL e as propriedades físicas da madeira, se destacando o E. saligna; 
Os melhores resultados de correlação entre DRL e as propriedades mecânicas foram obtidos para madeira de E. dunnii. Ao estratificar por idade, a mesma espécie aos 52 meses foi a única a apresentar uma alta correlação.

\section{AGRADECIMENTOS}

À Coordenação de Aperfeiçoamento de Pessoal de Nível Superior pela concessão da bolsa de estudo e a empresa GRANFLOR pela disponibilidade do material de estudo.

\section{REFERENCIAS BIBLIOGRÁFICAS}

BELTRAME, R. Desempenho silvicultural e seleção de clones de Eucalyptus spp. para a qualidade da madeira. 2012. 136 p. Tese (Doutorado em Engenharia Florestal) - Centro de Ciências Rurais, Universidade Federal de Santa Maria, Santa Maria, 2012.

CLAIR, B.; RUELLE, J.; THIBAUT, B. Relationship between growth stresses, mechanic physical properties and proportion of fibre with gelatinous layer in Chestnut (Castanea Sativa Mill.). Holzforschung, Berlin, v. 57, p. 189-195, 2003.

COPANT - COMISSÃO PANAMERICANA DE NORMAS TÉCNICAS. COPANT 459. Acondicionamiento de las maderas destinadas a los ensayos físicos y mecânicos. 1972a.

COPANT - COMISSÃO PANAMERICANA DE NORMAS TÉCNICAS. COPANT 461. Determinación Del peso especifico aparente. 1972b.

COPANT - COMISSÃO PANAMERICANA DE NORMAS TÉCNICAS. COPANT 462. Método de determinación de la contración.1972c.

DELUCIS, R. A.; MISSIO, A. L.; BELTRAME, R.; GATTO, D. A. Predição da deformação residual longitudinal por meio de propriedades dendrométricas e físicas de Acácia negra. Ciência da Madeira, Pelotas, v. 05, n. 01, p. 42-57, 2014.

FERNANDES, P. S. Variações de densidade da madeira e suas relações com as tensões de crescimento em progênie de Eucalyptus urophylla S. T. Blake. 1982, 85 p. Dissertação (Mestrado em Engenharia Florestal) Escola Superior de Agricultura "Luiz de Queiroz", Universidade de São Paulo, Piracicaba, 1982.

GONÇALVES, F. G.; OLIVEIRA, J. T. S.; SILVA, G. F.; NAPPO, M. E.; TOMAZELO FILHO, M. Parâmetros dendrométricos e correlações com propriedades tecnológicas em um híbrido clonal de Eucalyptus urophylla $\mathrm{x}$ Eucalyptus grandis. Revista Árvore, Viçosa, v. 34, n. 5, p. 947-959, 2010.

GONÇALVES, M. P. M. Correlações da altura e diâmetro com tensões de crescimento em árvores de Eucalyptus citriodora Hook e Eucalyptus urophylla S. T. Blake. 2007. 31 p. Monografia (Graduação em Engenharia Florestal) - Universidade Federal Rural do Rio de Janeiro, Seropédica, 2007.

IBGE - INSTITUTO BRASILEIRO DE GEOGRAFIA E ESTATÍSTICA. Mapa da vegetação do Brasil e Mapa de Biomas do Brasil. 2004. Disponível em . Acesso em: 30 nov. 2015.

LIMA, J. T.; TRUGILHO, P. F.; ROSADO, S. C. S.; CRUZ, C. R. Deformações residuais longitudinais decorrentes de tensões de crescimento em eucaliptos e suas associações com outras propriedades. Revista Árvore, Viçosa, v. 28, n. 1 , p. $107-119,2004$.

LIMA, L. L.; GARCIA, J. N.; NOGUEIRA, M. C. S. Influência do desbaste nas tensões de crescimento de Eucalyptus grandis Hill ex-Maiden. Scientia Forestalis, Piracicaba, n. 58, p. 111-125, 2000. 
Braz et al. - Relação entre a deformação residual longitudinal com

crescimento e propriedades da madeira de espécies de Eucalyptus

MALAN, F. S. Studies on the phenotypic variation in growth stress intensity and its association with tree and wood properties of South African grown Eucalyptus grandis. 1984, 272 p. Tese (Doutorado) - University of Stellenbosch, Stellenbosch, 1984.

MIRANDA, M. D. A.; NAHUZ, M. A. R. Estudo da influência do espaçamento de plantio de Eucalyptus saligna nos índices de rachamento após o desdobro e após a secagem. Scientia Florestalis, Piracicaba, n. 55, p. 107116, 1999.

MUNERI, A.; LEGATE, W.; PALMER, G. Relationships between surface growth strain and some tree wood and sawn timber characteristics of Eucalyptus cloeziana. Southern African Forestry Journal, Pretoria, v.187, p.4149, 1999.

NICHOLSON, J. E.; HILLIS, W. E.; DIRCHBURNE, N. Some tree growth: wood property relationships of eucalypts. Canadian journal of forest research, Ottawa, v. 5, n. 3, p. 424-432, 1975.

NUTTO, L. Manejo do crescimento diamétrico de Araucaria angustifolia (BERT.) O. KTZE. baseado na árvore individual. Ciência Florestal, Santa Maria, v. 11, n. 2, p. 9-25, 2001.

PÁDUA, F. A.; TRUGILHO, P. F.; ROSADO, S. C. S.; LIMA, J. T.; CARDOSO JR., A. A.; OLIVEIRA, A. C. Estimativa de parâmetros genéticos das tensões de crescimento em clones de Eucalyptus. Scientia Forestalis, Piracicaba, n. 66, p. 152-160, 2004.

SOUZA, M. A. M. Deformação Residual Longitudinal (DRL) causada pelas tensões de crescimento em clones de híbridos de Eucalyptus. 2002. 72 p. Dissertação (Mestrado em Ciência e Tecnologia da Madeira) Universidade Federal de Lavras, Lavras, 2002.

TRUGILHO, P.F.; LIMA, J.T.; MENDES, L.M. Influência da idade nas características do eucalipto. Revista da Madeira, Curitiba, n. 92, p. 82-88, 2005.

TRUGILHO, P. F.; IWAKIRI, S.; ROCHA, M. P.; MATOS, J. L. M.; SALDANHA, L. K. Efeitos da idade e classe diamétrica na deformação residual longitudinal em árvores de Eucalyptus dunnii Maiden. Revista Árvore, Viçosa, v. 28, n. 5, p. 725-731, 2004.

TRUGILHO, P. F.; LIMA. J. T.; PÁDUA, F. A.; SORAGI, L. C.; ANDRADE, C. R. Deformação residual longitudinal (DRL) e tangencial (DRT) em seis clones de Eucalyptus spp. Cerne, Lavras, v. 12, n. 3, p. 279-286, 2006.

TRUGILHO, P. F. ROSADO, S. C. S.; LIMA, J. T.; PÁDUA, F. A.; SOUZA, M. A. M. Deformação residual longitudinal (DRL) e sua relação com as características de crescimento da árvore de clones de Eucalyptus. Cerne, Lavras, v. 13, n. 2, p. 130-137, 2007.

WILKINS, A. P.; KITAHARA, R. Silvicultural treatments and associated growth rates, growth strains and wood properties in 12, 5-year-old Eucalyptus grandis. Australian Forestry, Melbourne, v. 54, n. 1-2, p. 99-104, 1991.

YANG, J. L., FIFE, D.; MATHESON, A. C. Growth strain in three provenances of plantation-grown Eucalyptus globulus Labill. Australian Forestry, Melbourne, v. 64, n. 4, p. 248-256, 2001.

Recebido em 09/07/2015

Aceito para publicação em 28/01/2016 